\title{
In vivo evaluation of DSAEK interface with scanning-laser confocal microscopy
}

\author{
Giulio Ferrari ${ }^{*}$, Verena Reichegger ${ }^{2}$, Luca Ludergnani ${ }^{2}$, Elisabetta Delfini ${ }^{2}$ and Claudio Macaluso ${ }^{2}$
}

\begin{abstract}
Background: Descemet Stripping Automated Endothelial Keratoplasty (DSAEK) allows selective replacement of the endothelium. Post-operative haze and particles can affect the interface quality and, ultimately, visual outcome. In this study, we evaluated DSAEK interface with in vivo laser confocal microscopy (LCM) in order to: (i) correlate interface status with best corrected visual acuity, and (ii) with time from surgery; (iii) correlate interface particle number with best corrected visual acuity. Host-donor interface was imaged and graded using a published reflectivity scale. Particles at the interface were counted.

Methods: 18 eyes of 16 patients ( 6 males and 10 females); mean age: $74 \pm 8.3$ years which underwent DSAEK were examined by means of in vivo laser confocal microscopy between 1 and 24 months after surgery. Host-donor interface was imaged and graded using a published reflectivity scale. Particles present at the interface were counted.

Results: Interface reflectivity was $2.17 \pm 1.2$ and significantly correlated with visual acuity (Spearman correlation coefficient $-0.83 ; P<0.001$ ), and with time after surgery (Spearman correlation coefficient $-0.87 ; P<0.001$ ). Visual acuity was $0.67 \pm 0.27$. The number of particles was $205 \pm 117.8$; no correlation was found between this number and visual acuity (Spearman correlation coefficient $-0.41 ; P=0.15$ ).

Conclusion: DSAEK interface imaged with LCM is helpful in diagnosing poor host-donor interface quality in DSAEK surgery. A good quality interface is related to a better visual acuity. Moreover, the quality of the interface appears to improve as time passes from the surgery. Interface quality is related with visual acuity and improves with time from surgery. LCM should be considered as an added tool in post-DSAEK follow-up of patients. Finally, our study shows that the presence of particles does not influence visual outcome.
\end{abstract}

Keywords: DSAEK, Interface, Laser corneal confocal microscopy

\section{Background}

Descemet Stripping Automated Endothelial Keratoplasty (DSAEK) is a recently developed lamellar corneal transplant which allows selective replacement of the endothelial side of the cornea. It has been proposed as an alternative to penetrating keratoplasty (PK) in cases of dysfunctional endothelium [1-3]. DSAEK has gained increasing popularity as it induces less post-operative astigmatism, less high order aberrations, and encounters higher patient satisfaction, when compared to PK [4]. Differently from PK, however, DSAEK creates a donorhost interface which can cause significant haze and

\footnotetext{
* Correspondence: giulio.ferrari@schepens.harvard.edu

${ }^{1}$ G.B. Bietti Eye Foundation, IRCCS, Rome, Italy

Full list of author information is available at the end of the article
}

possibly influence visual recovery. Moreover, particles of different nature (lint, cellular debris, plastic or metallic particles) can be detected often times at the slit-lamp and their possible impact on visual acuity has been object of concern [5].

A new generation of in-vivo laser confocal microscopy devices (Heidelberg Retina Tomograph 2 Rostock Cornea Module; Heidelberg Engineering $\mathrm{GmbH}$, Dossenheim, Germany) has become available recently [6-8]. These instruments provide histology-quality images, without the need to remove, stain and cut the tissue, with a higher axial resolution $(4 \mu \mathrm{m})$ than that achieved with white-light confocal microscopy $(10 \mu \mathrm{m}$ with ConfoScan 2, Nidek Technologies, Vigonza, Italy). This case series investigates the impact of interface reflectivity and

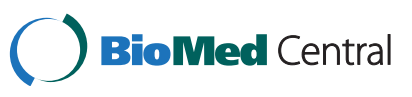


particles on visual acuity in 16 patients at different timepoints. Patients were consecutively enrolled during a two-month time frame.

\section{Methods}

The ophthalmology department board approved this study. Informed consent was obtained prior to performing confocal microscopy; the study followed the tenets of the Declaration of Helsinki. 18 eyes of 16 patients (6 males and 10 females) who underwent DSAEK surgery due to Fuchs' dystrophy at the University Hospital of Parma, Italy were included in the study. Patients were proposed to join the study and the protocol was explained.

Exclusion criteria for this study were glaucoma, ocular infections and uveitis. Patients were imaged between 1 and 24 months following DSAEK surgery.

All patients underwent a complete ophthalmology visit including refraction with determination of ETDRSmeasured Best Spectacle Corrected Visual Acuity (BSCVA), biomicroscopy, intraocular pressure measurement, and in-vivo scanning laser confocal microscopy (LCM).

Patients underwent DSAEK surgery alone or associated with cataract extraction (Table 1).

\section{DSAEK surgery}

All procedures were performed under monitored anesthesia with peribulbar block, following a published

Table 1 Demographics of the study population

\begin{tabular}{|c|c|c|c|c|c|}
\hline Patient & Age & Gender & $\begin{array}{l}\text { Gender } \\
\text { Diagnosis }\end{array}$ & Procedure & $\begin{array}{l}\text { BSCVA } \\
\text { (Snellen) }\end{array}$ \\
\hline 1 & 83 & M & Fuchs' & DSAEK & 1 \\
\hline 2 & 56 & $M$ & Fuchs' & DSAEK & 0.8 \\
\hline 3 & 78 & $\mathrm{~F}$ & Fuchs'+ cataract & $\mathrm{DSAEK}+\mathrm{FACO}+\mathrm{IOL}$ & 0.6 \\
\hline 4 & 72 & $\mathrm{~F}$ & Fuchs' & DSAEK & 0.8 \\
\hline $5^{*}$ & 65 & $M$ & Fuchs' + cataract & $\mathrm{DSAEK}+\mathrm{FACO}+\mathrm{IOL}$ & 1 \\
\hline $6^{*}$ & 65 & $M$ & Fuchs' & DSAEK & 0.8 \\
\hline $7 *$ & 72 & $\mathrm{~F}$ & Fuchs' & DSAEK & 0.8 \\
\hline $8^{*}$ & 72 & $\mathrm{~F}$ & Fuchs'+ cataract & $\mathrm{DSAEK}+\mathrm{FACO}+\mathrm{IOL}$ & 0.8 \\
\hline 9 & 77 & $M$ & Fuchs' & DSAEK & 1 \\
\hline 10 & 85 & $\mathrm{~F}$ & Fuchs' & DSAEK & 0.6 \\
\hline 11 & 69 & $\mathrm{~F}$ & Fuchs' & DSAEK & 0.8 \\
\hline 12 & 82 & $F$ & Fuchs' & DSAEK & 0.4 \\
\hline 13 & 78 & $F$ & Fuchs' & DSAEK & 0.13 \\
\hline 14 & 77 & $\mathrm{~F}$ & Fuchs' & DSAEK & 0.4 \\
\hline 15 & 83 & $\mathrm{~F}$ & Fuchs' & DSAEK & 0.2 \\
\hline 16 & 68 & $F$ & Fuchs' & DSAEK & 1 \\
\hline 17 & 85 & $M$ & Fuchs' & DSAEK & 0.4 \\
\hline 18 & 65 & $M$ & Fuchs' & DSAEK & 0.6 \\
\hline
\end{tabular}

Asterisks indicate eyes from the same patient $(5,6$ and 7,8$)$. technique [9]. Briefly, donor lenticules were prepared with a Moria artificial chamber, a Carriazo-Barraquer microkeratome (300 $\mu \mathrm{m}$ microkeratome head), and a punching block (Moria, Antony, France). The anterior chamber of the recipient eye was then entered through a 4 to $5 \mathrm{~mm}$ clear corneal incision. An anterior chamber maintainer was used to prevent anterior chamber collapse during surgery. Descemet membrane was stripped from the central $8 \mathrm{~mm}$. The peripheral edge of the rolled endothelial graft was grasped from a device inserted in the corneal tunnel and pulled inside the anterior chamber with either coaxial forceps or a Prolene 10-0 suture. A small air bubble was injected to lift the donor tissue. After centering the graft, the anterior chamber was completely filled with air. After 10 minutes, the air bubble was reduced to about $80 \%$ of the size of the endothelial graft.

\section{In vivo confocal microscopy}

All eyes were examined using the same LCM device (Heidelberg Retina Tomograph 2 Rostock Cornea Module; Heidelberg Engineering $\mathrm{GmbH}$, Dossenheim, Germany). Briefly, patients were instilled with a drop of topical anesthetic (Oxybuprocaine chloridrate 0.4\%) 5 minutes and immediately before the exam. The patient was then asked to steadily fixate a target and LCM was performed on the central cornea as we described previously [10]. The exam lasted approximately 10 minutes. At the end of the examination, antibiotic ointment (Ofloxacin 0.3\%) was applied.

Examination was performed taking scans of the epithelium, and progressively focusing to deeper layers down to the interface area. Interface area was identified as the acellular zone found proceeding from the epithelium to the endothelium. This area hosted often times birefringent particles, as previously described (11). At least three scans - chosen for image quality - were considered. Images obtained were 400x400 microns wide. Particle density was measured using a built-in software (Cornea section cell count; Heidelberg Engineering), which calculates the density of objects manually selected by the operator. In order to minimize variability, a single investigator blinded to the patient group analyzed and graded all the images retrospectively. Images were graded following a published method specifically studied for HRT2 confocal microscope [11]. The grading was performed by comparing our images with sample images provided by Kobayashi et al. We used a 4 grade scale (grade 1: no haze; grade 4: severe haze).

\section{Statistical analysis}

Data analysis was performed using SPSS for Windows (version 11.0, SPSS Inc, Chicago, Illinois). Correlation was studied by means of Spearman Correlation 
Coefficient. Difference was considered statistically significant if the $P$ value was $<0.05$, and highly significant if $\mathrm{P}$ was $<0.01$.

\section{Results and discussion}

We examined 6 males and 10 females (16 patients, 18 eyes). The mean age was $74 \pm 8.3$ and ranged from 56 to 85 years. Table 1 provides the demographics of the study population.

Interface reflectivity was $2.17 \pm 1.2$ and significantly correlated with visual acuity (Spearman correlation coefficient $-0.83 ; \mathrm{P}<0.001$ ), and with time after surgery (Spearman correlation coefficient -0.87 ; $\mathrm{P}<0.001$ ); Figure $1 \mathrm{~A}$ and $\mathrm{B}$.

Visual acuity averaged over all the patients was $0.67 \pm 0.27$. The number of particles was $205 \pm 117.8$. No correlation was found between the number of particles at the interface and visual acuity (Spearman correlation coefficient $-0.41 ; \mathrm{P}=0.15$ ); Figure $1 \mathrm{C}$.

Moreover, we did not find a significant correlation between the time from surgery and the number of interface particles (Spearman coefficient 0.53, $\mathrm{P}=0.06$ ).

Interface quality assessment with slit-lamp and LCM appeared consistent (Figure 2).

DSAEK surgery is considered a valuable alternative to PK in cases of dysfunctional endothelium such as in Fuchs' dystrophy as it selectively replaces it. DSAEK is less invasive and better tolerated by patients [4], allows prompt improvement and stability of visual acuity. However, being a lamellar surgery, it creates an interface between the donor and the recipient tissue, where the healing process is thought to be critical to optimal visual recovery [11]. DSAEK grafts present increased haze at least up to three months, when compared to PK grafts [12]. Loss of corneal transparency and swelling in Fuch's dystrophy have been object of extensive studies [13].

The most common cause of DSAEK failures is secondary to endothelial cell loss, followed by "dysfunctional" donor-host interface. These include lenticule dislocation, interface fibrosis or hemorrhage, epithelial downgrowth [14-16].

LCM is a low-invasivity, repeatable method which allows to study the interface histology in vivo, and hence is suitable to study the interface healing process.

Previous studies have evaluated the interface on a smaller sample of patients, or at lower resolution with white-light confocal microscopy. Our study shows a correlation between visual acuity and interface grading. Interestingly, the deep part of the stroma, where DSAEK surgery takes place, is interested by a number of pathological changes. For example, it has been shown that fluid entering the cornea causes more swelling in the posterior than in the anterior lamellae. Also, posterior lamellae can reach higher degree of final hydration, and collagen-free regions (known as "lakes") exist in Fuch's dystrophy corneas which are thought to be caused by dead cells and fibril disordering. The selective distribution of fluid into the corneal stroma might reflect a different glycosaminglycan concentration in the posterior part of the cornea [13]. All these observations contribute to explain the formation of an interface haze following DSAEK, and corroborate a correlation between haze extent and visual acuity loss. To our knowledge, this is the

A

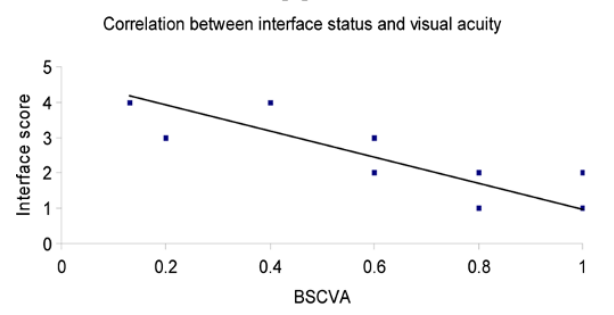

B

ind trom

Correlation between interface
surgery

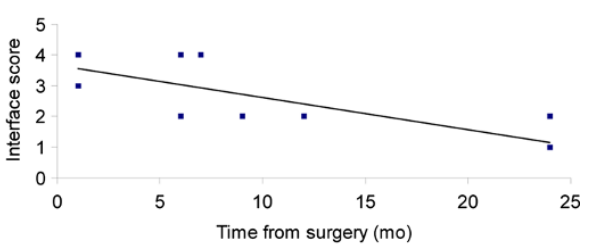

C

Correlation between particle number and visual acuity

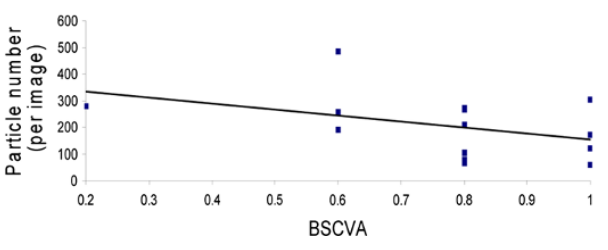

Figure 1 A. Negative correlation between interface reflectivity and BSCVA. The higher the interface reflectivity, the lower the visual acuity (Spearman correlation coefficient $-0.83 ; \mathrm{P}<0.001)$ B. Negative correlation between interface reflectivity and with time after surgery (Spearman correlation coefficient $-0.87 ; \mathrm{P}<0.001$ ). The longer the time from surgery, the lower the interface reflectivity. $\mathbf{C}$. No correlation was found between the number of particles at the interface and visual acuity (Spearman correlation coefficient $-0.41 ; \mathrm{P}=0.15$ ). 

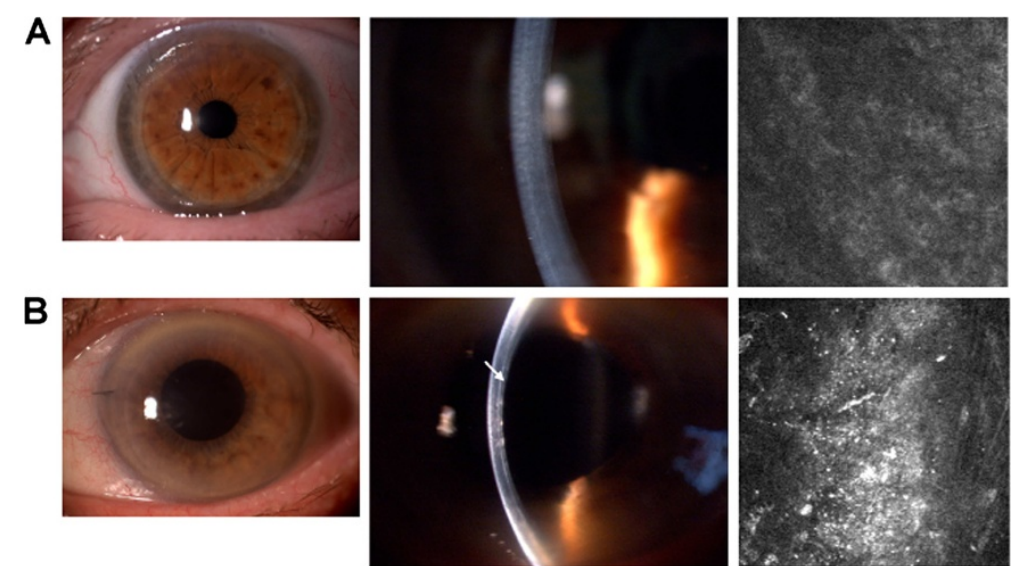

Figure 2 Panel A. Representative slit lamp and confocal pictures of a low reflectivity (i.e. good quality) interface. Note the clear interface observed at the slit lamp, which corresponds to a dark confocal image. The BSCVA in this patient was 20/20. Panel B. Representative slit lamp and confocal pictures of a high reflectivity (i.e. poor quality) interface. Note the evident white line representing the donor-host interface (arrow), which corresponds to a hazy confocal picture. The BSCVA in this patient was 20/40.

first report finding a correlation between a published confocal HRT2 measure and BSCVA. This finding is in contrast with what previously reported by Espana et al. [17], using a different microscope (ConfoScan as opposed to HRT). However, caution should be used in comparing these two studies, as the axial resolution provided by HRT is more than double than the ConfoScan, and this could explain the different findings.

Similarly to others $[17,18]$, we found no correlation between particle number and visual acuity. Small particles located at the interface are a common finding following DSAEK, and are also observed after LASIK surgery [19]. The origin of interface particles has been object of research: they are generally thought to be associated with the use of the microkeratome [20-22], but they were also observed after femtosecond laser application [23]. Although previous studies suggested a deleterious effect of particles on visual acuity [5], it is now becoming clear that this may not be the case $[17,18]$. Our study confirms that small debris should not represent a concern to the surgeon. Interestingly, we did not find any correlation between the particle number and time from surgery (Spearman correlation coefficient 0.53 , even though the P value (0.06) was not extremely low). In contrast with this, Kobayashi et al. reported a progressive reduction of particle number with time $[11,24]$. Our findings could also be due to a difference in the materials used and/or environment encountered during the surgery and/or the relatively small size of our sample. Similarly to other authors, we did not find activated keratocytes or dendritic cells at the interface $[11,17,24]$. This could be due to either the low density of these cells in the posterior stroma, or to the time passed from surgery, which would allow keratocytes to become quiescent.
A correlation between interface grading and time form surgery- suggested by this study- was confirmed by other papers $[11,24]$ implying that a healing process occurring at the interface - an actual surgical wound in the cornea - is associated with a progressive gain in visual acuity.

We would like to point out that this study also had limitations. One of these is represented by the small sample number; hence bigger samples would be needed to confirm our findings. Secondarily, other potentially useful outcomes such as topography and pachymetry were not considered. We anticipate this may be the object of future studies.

\section{Conclusions}

In the present study, we quantified the interface quality following DSAEK surgery. We found that a good quality interface is related to a better visual acuity, and improves with time from surgery. Also, the presence and number of particles imaged at the interface is not related with visual outcome. We propose that in vivo LCM could be a helpful tool in the follow-up of DSAEK patients, as the study of the interface gives information potentially relevant for major clinical outcomes (such as BSCVA) together with other relevant information, such as endothelial cell count and keratocyte activation status.

\section{Informed consent}

This study was performed with informed consent and in accordance with institutional guidelines.

\section{Competing interests}

No financial and non-financials interests to disclose.

\section{Authors' contributions}

GF imaged the patients participated in image grading and drafted the manuscript. VR participated in patient recruitment. LL participated in patient recruitment and patient management. ED participated in patient imaging. 
$\mathrm{CM}$ is the senior author and this study was started and completed under his direct supervision, he coordinated the study design and helped to draft the manuscript. All authors read and approved the final manuscript.

\section{Source(s) of funding}

Giulio Ferrari received a research grant from the Bietti Eye Foundation, IRCCS Rome, Italy. Claudio Macaluso receieved a grant from Programma di Ricerca Regione Università (DGR22422007)

Other authors have no sources of funding to declare.

\section{Author details}

'G.B. Bietti Eye Foundation, IRCCS, Rome, Italy. ${ }^{2}$ Dept. of Ophthalmology, University Hospital of Parma, Parma, Italy.

Received: 5 March 2012 Accepted: 29 June 2012

Published: 1 August 2012

\section{References}

1. Chen ES, Terry MA, Shamie N, Chen ES, Terry MA, Shamie N, Hoar KL, Friend DJ: Descemet-stripping automated endothelial keratoplasty: six-month results in a prospective study of 100 eyes. Cornea 2008, 27: 514-520.

2. Koenig SB, Covert DJ: Early results of small-incision Descemet's stripping and automated endothelial keratoplasty. Ophthalmology 2007, 114: 221-226.

3. Koenig SB, Covert DJ, Dupps WJ Jr, Meisler DM: Visual acuity, refractive error, and endothelial cell density six months after Descemet stripping and automated endothelial keratoplasty (DSAEK). Cornea 2007, 26: 670-674.

4. Bahar I, Kaiserman I, Levinger E, Sansanayudh W, Slomovic AR, Rootman DS: Retrospective contralateral study comparing descemet stripping automated endothelial keratoplasty with penetrating keratoplasty. Cornea 2009, 28:485-488.

5. Kaufman SC, Musch DC, Belin MW, Cohen EJ, Meisler DM, Reinhart WJ, Udell IJ, Van Meter WS: Confocal microscopy: a report by the American Academy of Ophthalmology. Ophthalmology 2004, 111:396-406.

6. Stave J, Zinser G, Grümmer G, Guthoff R: Modified Heidelberg Retinal Tomograph HRT. Initial results of in vivo presentation of corneal structures. Ophthalmologe 2002, 99:276-280.

7. Kobayashi A, Yokogawa H, Sugiyama K: In vivo laser confocal microscopy of Bowman's layer of the cornea. Ophthalmology 2006, 113:2203-2208.

8. Kobayashi A, Sugiyama K: In vivo laser confocal microscopy findings for Bowman's layer dystrophies (Thiel-Behnke and Reis-Bucklers corneal dystrophies). Ophthalmology 2007, 114:69-75.

9. Macaluso C: Closed-chamber pulling-injection system for donor graft insertion in endothelial keratoplasty. J Cataract Refract Surg 2008, 34: 353-356.

10. Ferrari G, Tedesco S, Delfini E, Macaluso C: Laser Scanning In Vivo Confocal Microscopy in a Case of Terrien Marginal Degeneration. Cornea 2010, 29 (4):471-475.

11. Kobayashi A, Mawatari Y, Yokogawa H, Sugiyama K: In vivo laser confocal microscopy after descemet stripping with automated endothelial keratoplasty. Am J Ophthalmol 2008, 145:977-985.

12. Uchino $Y$, Shimmura S, Yamaguchi T, Kawakita T, Matsumoto Y, Negishi $K$, Tsubota K: Comparison of corneal thickness and haze in DSAEK and penetrating keratoplasty. Cornea 2011, 30(3):287-290.

13. Meek KM, Leonard DW, Connon CJ, Dennis S, Khan S: Transparency, swelling and scarring in the corneal stroma. Eye (Lond) 2003, 17(8): 927-936.

14. Shulman J, Kropinak M, Ritterband DC, Perry HD, Seedor JA, McCormick SA, Milman T: Failed descemet-stripping automated endothelial keratoplasty grafts: a clinicopathologic analysis. Am J Ophthalmol 2009, 148(e2): 752-759.

15. Oster SF, Ebrahimi KB, Eberhart CG, Schein OD, Stark WJ, Jun AS: A clinicopathologic series of primary graft failure after Descemet's stripping and automated endothelial keratoplasty. Ophthalmology 2009, 116:609-614.

16. Sbarbaro JA, Eagle RC, Thumma P, Raber IM: Histopathology of posterior lamellar endothelial keratoplasty graft failure. Cornea 2008, 27:900-904.
17. Espana EM, Huang B: Confocal microscopy study of donor-recipient interface after Descemet's stripping with endothelial keratoplasty. $\mathrm{Br} J$ Ophthalmol 2010, 94:903-908.

18. Prasher P, Muftuoglu O, Bowman RW, McCulley JP, Petroll WM, Cavanagh HD, Mootha W: Tandem scanning confocal microscopy of cornea after descemet stripping automated endothelial keratoplasty. Eye Contact Lens 2009, 35:196-202.

19. Kaufman SC, Kaufman HE: How has confocal microscopy helped us in refractive surgery? Curr Opin Ophthalmol 2006, 17:380-388

20. Perez-Gomez I, Cameron I, Efron N: Particles at the laser in situ keratomileusis flap interface. J Cataract Refract Surg 2004, 30:2021.

21. Dawson DG, Edelhauser HF, Grossniklaus HE: Long-term histopathologic findings in human corneal wounds after refractive surgical procedures. Am J Ophthalmol 2005, 139:168-178.

22. Ivarsen A, Thøgersen J, Keiding SR, Hjortdal JØ, Møller-Pedersen T: Plastic particles at the LASIK interface. Ophthalmology 2004, 111:18-23.

23. Hu MY, McCulley JP, Cavanagh HD, Bowman RW, Verity SM, Mootha W, Petroll WM: Comparison of the corneal response to laser in situ keratomileusis with flap creation using the FS15 and FS30 femtosecond lasers: clinical and confocal microscopy findings. I Cataract Refract Surg 2007, 33:673-681.

24. Kobayashi A, Yokogawa H, Sugiyama K: In vivo laser confocal microscopy after non-Descemet's stripping automated endothelial keratoplasty. Ophthalmology 2009, 116:1306-1313.

doi:10.1186/1471-2415-12-32

Cite this article as: Ferrari et al: In vivo evaluation of DSAEK interface with scanning-laser confocal microscopy. BMC Ophthalmology 2012 12:32.

\section{Submit your next manuscript to BioMed Central and take full advantage of:}

- Convenient online submission

- Thorough peer review

- No space constraints or color figure charges

- Immediate publication on acceptance

- Inclusion in PubMed, CAS, Scopus and Google Scholar

- Research which is freely available for redistribution 